\title{
TaqIB polymorphism in CETP gene: the influence on incidence of cardiovascular disease in statin-treated patients with familial hypercholesterolemia
}

\author{
Martina F Mohrschladt ${ }^{1}$, Femke van der Sman-de Beer ${ }^{2}$, Maaike K Hofman ${ }^{3}$, \\ Marieke van der Krabben ${ }^{1}$, Rudi GJ Westendorp ${ }^{1}$ and August HM Smelt*,1 \\ ${ }^{1}$ Department of General Internal Medicine, Leiden University Medical Center, PO Box 9600, 2300 RC Leiden,
The Netherlands; ${ }^{2}$ Department of Clinical Epidemiology, Leiden University Medical Center, PO Box 9600,
2300 RC Leiden, The Netherlands; ${ }^{3}$ Gaubius Laboratory, TNO-PG, Leiden, The Netherlands
}

The effects of Taql restriction fragment length polymorphism of the CETP gene on the occurrence of cardiovascular disease (CVD) events were investigated in patients with familial hypercholesterolemia (FH). A total of $300 \mathrm{FH}$ patients, of which $116(39 \%)$ had CVD at the start of the study, were treated with statins during a mean period of 8.5 years. The distribution of Taq1B genotypes was $31 \%$ B1B1, 49\% B1B2, and $20 \%$ B2B2. No differences were found at baseline between the three genotypes, except for an association of the B1 allele with lower high-density lipoprotein $(H D L)$-cholesterol levels $(P=0.003)$. All patients were put on statins within 6-8 weeks after the first visit; about $60 \%$ received simvastatin (20-40 mg daily) and $40 \%$ either pravastatin ( $40 \mathrm{mg}$ daily) or atorvastatin $(20-40 \mathrm{mg}$ daily). The different statin treatments were similar for all groups. The mean change of plasma HDL-cholesterol, low-density lipoproteincholesterol, and triglyceride concentration during statin therapy was similar for the three genotypes. During follow-up, new CVD events were recorded in $22(37 \%)$ of the B2B2 patients $(n=59)$ and in 67 (28\%) of B1 allele carriers $(n=241)(P=0.36)$. The relative risk for CVD events, after adjustment for age, gender, and CVD at intake, was 1.8 (Cl: 1.1-3.0) for B2B2 carriers compared to B1 allele carriers. The Taq1B polymorphism is a significant predictor of future CVD events in statin-treated patients with $\mathrm{FH}$. In spite of similar improvement of the lipoprotein profile during statin therapy, our FH patients with the B2B2 genotype may have a higher CVD risk in comparison with the B1 allele carriers.

European Journal of Human Genetics (2005) 13, 877-882. doi:10.1038/sj.ejhg.5201426

Published online 27 April 2005

Keywords: familial hypercholesterolemia; CETP; polymorphism; cardiovascular disease; TaqIB; prospective follow-up; statins

\section{Introduction}

High levels of plasma high-density lipoprotein (HDL)cholesterol are associated with a decreased risk of cardiovascular disease (CVD). ${ }^{1,2}$ Increased activity of plasma

*Correspondence: AHM Smelt, Department of General Internal Medicine, Leiden University Medical Center, PO Box 9600, 2300 RC Leiden, The Netherlands. Tel: + 3171 5264494; Fax: + 3171 5248159;

E-mail: a.h.m.smelt@lumc.nl

Received 13 August 2004; revised 14 March 2005; accepted 16 March 2005 cholesteryl ester transfer protein (CETP), which facilitates the reverse cholesterol transport of cholesteryl ester from the HDL fraction to triglyceride (TG)-rich lipoproteins, is negatively related to the level of plasma HDL-cholesterol. CETP deficiencies, due to mutations in the CETP gene, which is located on chromosome $16 \mathrm{q} 21$, are associated with high levels of HDL-cholesterol, ${ }^{3}$ and therefore might be related to longevity. Several studies report on the association between plasma HDL-cholesterol concentrations and the restriction fragment length polymorphism 
(RFLP) in intron 1 of the CETP gene. ${ }^{4-8}$ The presence or absence of a TaqI restriction site leads to three possible genotypes for this TaqI RFLP: B1B1, B1B2, and B2B2. Individuals with B1B1 (homozygous for the presence of the restriction site) have lower plasma HDL-cholesterol levels, compared to carriers of the B2 allele.

Patients with familial hypercholesterolemia (FH) are especially at risk for the development of premature CVD. ${ }^{9}$ However, the occurrence of CVD in FH patients varies depending on additional genetic and environmental factors. ${ }^{10,11}$ Therefore, this study was initiated to investigate the influence of the TaqIB polymorphism on CVD in patients with $\mathrm{FH}$, and to assess whether the CETP TaqIB polymorphism can predict the development of new or recurrent cardiovascular events in these patients.

\section{Patients and methods Patients}

From a total sample of $400 \mathrm{FH}$ patients, who received and responded to a questionnaire (response rate 96\%) on cardiovascular events after intake at the Lipid Clinic from the Leiden University Medical Center, 300 statin-treated patients (143 male and 157 female patients) of whom a DNA sample was available, were included in the present study. Details of the methods were described earlier. ${ }^{12} \mathrm{~A}$ total of 100 patients were excluded for the following reasons: from 57 subjects there was no DNA sample available, 39 subjects were not treated with statins, and four subjects were excluded because of first-degree relationship to an index patient. Age ranged from 15 to 72 years (mean age 48 years). At intake, the medical history was taken, as well as information about risk factors for CVD and the presence of premature CVD in the family. The family history was considered positive if one of the patients' firstdegree relatives had manifest CVD before the age of 50 years. All patients underwent a routine physical examination. Diagnosis of FH was based on the following criteria: (1) a repeated serum cholesterol $>8.0 \mathrm{mmol} / \mathrm{l}$ and TG levels $<2.5 \mathrm{mmol} / \mathrm{l}$ (the mean value of two fasting blood samples obtained with an interval of 3 weeks), and (2) a family history of hypercholesterolemia and/or premature CVD or (3) the presence of tendon xanthomas. Exclusion criteria for $\mathrm{FH}$ were secondary hyperlipidemia due to diabetes mellitus or renal, liver, or thyroid disease. CVD was present at baseline in $39 \%$ of the patients. The criteria for having CVD at intake were a medical history of one or more of the following: angina pectoris (AP), myocardial infarction (MI), intermittent claudication (CI), percutaneous transluminal coronary angioplastic (PTCA), coronary artery bypass grafting (CABG), and arterial peripheral vascular surgery. A cardiovascular event during the followup period was designated as newly diagnosed $\mathrm{AP}, \mathrm{MI}$, arterial peripheral vascular disease, PTCA and/or CABG, and death from CVD. All information on events was confirmed by tracking hospital records. In a similar way, we obtained information from hospital records of the deceased patients. The use of statin medication (type of drug, dosage, and initiation date) was assessed by the questionnaire, and medical as well as pharmacy records confirmed all information given by the patients. The local hospital ethics committee approved the study and informed consent was obtained from all participants.

\section{Blood sampling}

All blood samples were collected after an overnight fast early in the morning. Serum was obtained after centrifugation at $1200 \mathrm{~g}$ for $10 \mathrm{~min}$ at room temperature and stored at $-20^{\circ} \mathrm{C}$ until use. Samples for DNA analysis were isolated according to standard procedures and stored at $4^{\circ} \mathrm{C}$ until use.

\section{Lipids and lipoproteins}

Total serum cholesterol, TG, and HDL-cholesterol levels were determined using enzymatic assay kits (Boehringer, Mannheim, Germany), whereas low-density lipoprotein (LDL)-cholesterol was calculated by the Friedewald equation. ${ }^{13}$

\section{DNA analysis}

DNA was isolated from peripheral blood leukocytes by standard methods. ${ }^{14}$ The TaqIB polymorphism in intron 1 of the CETP gene was detected by a polymerase chain reaction (PCR)-based method using primers 5'-CAC ACC ACT GCC TGA TAA CC-3' (sense) and 5'-GTG ACC CCC AAC ACC AAA TA-3' (antisense). The amplification mixture included $25 \mathrm{pmol}$ of each primer, $100 \mathrm{ng}$ genomic DNA, $0.2 \mathrm{mmol} / \mathrm{l}$ of each dNTP, $10 \mathrm{mmol} / \mathrm{l}$ Tris- $\mathrm{HCl}(\mathrm{pH}$ 9.0), $1.5 \mathrm{mmol} / \mathrm{l} \mathrm{MgCl}_{2}, 50 \mathrm{mmol} / \mathrm{l} \mathrm{KCl}, 0.01 \%$ (w/v) gelatin, $0.1 \%$ Triton X-100, $80 \mu \mathrm{g} / \mathrm{ml} \mathrm{BSA}$, and $0.2 \mathrm{U}$ Taq polymerase (Super Taq, HT Biotechnology Ltd, Cambridge, UK) in a total volume of $50 \mu \mathrm{l}$. Amplification was performed for 32 cycles of $1 \mathrm{~min}$ at $94^{\circ} \mathrm{C}, 30 \mathrm{~s}$ at $57^{\circ} \mathrm{C}$, and $1.5 \mathrm{~min}$ at $72^{\circ} \mathrm{C}$ with an initial denaturation period of 4 min. About $20 \mu \mathrm{l}$ of PCR products was digested with the restriction enzyme TaqI according to the recommendations of the supplier (Life Technologies). Fragments were separated on a $2 \%$ MP agarose gel (Boehringer Mannheim, Germany) and stained with ethidium bromide. One fragment of $505 \mathrm{bp}$ indicated the absence of the TaqI restriction site (B2B2 genotype), two fragments of 415 and $90 \mathrm{bp}$ indicated the presence of the restriction site (B1B1), and three fragments of 505,415 , and $90 \mathrm{bp}$ indicated heterozygosity for the restriction site (B1B2).

\section{Statistical analysis}

Continuous data are presented as means $( \pm S D)$, unless stated otherwise. Differences in baseline characteristics and concentrations of lipids and lipoproteins between the TaqI polymorphic forms of the CETP gene were tested by one- 
way analysis of variance (ANOVA) or Pearson's $\chi^{2}$ test for frequencies. Changes in lipid parameters after statin treatment were expressed as means $( \pm S D)$, and the differences within groups were tested with paired samples $t$-test, while the differences between groups were analyzed by ANOVA. The Hardy-Weinberg equilibrium was calculated by gene counting and $\chi^{2}$ analysis.

Cumulative event-free survival was analyzed with the Kaplan-Meier method and with Cox's regression. Survival time was defined as the period from the date of intake at the lipid clinic to the date of first cardiovascular event or death or to the end of the study (ie the day that the participant responded to the questionnaire). The COX regression model used for this analysis of the CETP polymorphism as a risk factor for future cardiovascular events was adjusted for age, gender, the presence of CVD at intake, and baseline lipid parameters. Differences were considered significant at $P<0.05$. Statistical analyses were performed with SPSS/PC $+{ }^{\mathrm{TM}}$ software (SPSS, Chicago, IL, USA).

\section{Results}

\section{Genotype frequencies}

The presence and absence of a restriction site for TaqI in intron 1 of the CETP gene was denoted as B1 and B2, respectively. The following distribution of TaqIB genotypes was found: $31 \%(n=93)$ of the patients were homozygous for the presence of the restriction site (B1B1), whereas $49 \%$ $(n=148)$ were heterozygous (B1B2), and 20\% $(n=59)$ were homozygous for the absence of the restriction site (B2B2). The gene frequencies for the TaqIB polymorphism were 0.557 for the B1 allele and 0.443 for the B2 allele. The observed allele frequencies were in Hardy-Weinberg equilibrium.

\section{Baseline characteristics and associations with lipid levels}

Overall, 39\% ( $n=116)$ of the patients had (a history of) CVD when entering the lipid clinic. Patient baseline characteristics and lipid profiles are shown in Table 1. There was no difference in the distribution of gender and the presence of CVD at intake or positive family history for CVD in the different genotypes. While there was a significant association of the B1 allele with lower HDLcholesterol concentrations (ANOVA $P=0.003$ ), there were no significant differences in BMI, total cholesterol (Tcholesterol), and LDL-cholesterol levels between the different genotypes. Patients homozygous for the B1 allele tended to have lower levels of serum TG compared to carriers of two different alleles $(P=0.06)$. There was an influence of smoking habits with the association of the genotypes and HDL-cholesterol. Analysis of this association in three groups (never smokers, ever smokers, and current smokers) showed that only in subjects who had never smoked, there was a significantly lower mean plasma HDL level in the B1 allele carriers than in the B2B2 subjects $(1.27 \pm 0.34$ vs $1.47 \pm 0.36 \mathrm{mmol} / \mathrm{l} ; P=0.02)$.

\section{Response to statin treatment}

The mean follow-up period for all 300 subjects was 8.5 years and similar for all three genotypes. All patients were put on statin medication for 6-8 weeks after the first visit to the lipid clinic. About $60 \%$ of the patients were treated with simvastatin (20-40 mg daily), and 40\% were treated with either pravastatin (40 mg daily) or atorvastatin (20$40 \mathrm{mg}$ daily). The average dosage and type of statin were similarly distributed in all three groups. Of all statintreated patients, $89 \%$ were continuously on medication, defined as the total period without statin medication less than 12 weeks. The remainder of the patients had periods not on statin medication amounting to 12 weeks and over. Although all 300 patients were treated with statins, a second blood sample for analysis of the effect of statin treatment was available only in 276 patients. This sample was taken after a period of 1 or 2 years after start of lipidlowering medication.

Paired analysis of lipid parameters showed that overall serum levels of T-cholesterol decreased by 31\%, LDLcholesterol by $39 \%$, and TG by $13 \%$, while the level of

Table 1 Baseline characteristics of $300 \mathrm{FH}$ patients according to the Taq1B polymorphism

\begin{tabular}{|c|c|c|c|c|}
\hline & $B 1 B 1(n=93)$ & $B 1 B 2(n=148)$ & $B 2 B 2(n=59)$ & $P$ \\
\hline No. of males $n(\%)$ & $48(52)$ & $70(47)$ & $25(42)$ & NS \\
\hline Age mean (range) (years) & $47(18-72)$ & $48(15-72)$ & $50(21-69)$ & NS \\
\hline T-cholesterol $(\mathrm{mmol} / \mathrm{l})$ & $9.69(1.78)$ & $9.86(1.70)$ & $9.96(1.73)$ & NS \\
\hline $\mathrm{TG}(\mathrm{mmol} / \mathrm{l})$ & $1.68(0.63)$ & $1.84(0.66)$ & $1.78(0.67)$ & NS \\
\hline HDL-cholesterol (mmol/l) & $1.17(0.30)$ & $1.23(0.32)$ & $1.35(0.35)$ & 0.003 \\
\hline LDL-cholesterol ( $\mathrm{mmol} / \mathrm{l})$ & $7.77(1.82)$ & $7.80(1.77)$ & $7.81(1.83)$ & NS \\
\hline $\mathrm{BMI}\left(\mathrm{kg} / \mathrm{m}^{2}\right)$ & $24.77(3.25)$ & $24.95(3.34)$ & $24.90(3.45)$ & NS \\
\hline Current smokers, $n(\%)$ & $32(34)$ & $53(36)$ & $16(27)$ & NS \\
\hline Fam. history CVD, $n(\%)$ & $40(43)$ & $57(39)$ & $24(41)$ & NS \\
\hline CVD at intake, $n(\%)$ & $36(39)$ & $60(41)$ & $20(34)$ & NS \\
\hline
\end{tabular}

Lipid parameters and BMI are shown as mean (SD). Frequencies are tested with $\chi^{2}$ test. Age, BMI, and lipid parameters are tested with ANOVA. 
Table 2 Changes in lipid and lipoprotein concentrations in $276 \mathrm{FH}$ patients after statin treatment

\begin{tabular}{lccr}
\hline & $B 1 B 1(n=89)$ & $B 1 B 2(n=131)$ & $B 2 B 2(n=56)$ \\
\hline Decrease in cholesterol & $2.99(1.35)$ & $3.07(1.47)$ & $3.09(1.56)$ \\
Decrease in TG & $0.18(0.63)$ & $0.26(0.64)$ & $0.22(0.54)$ \\
Increase in HDL & $0.13(0.24)$ & $0.14(0.26)$ & $0.08(0.19)$ \\
Decrease in LDL & $3.04(1.36)$ & $3.07(1.37)$ & $3.07(1.54)$ \\
\hline
\end{tabular}

Values are means (SD) for all lipids and lipoproteins.

${ }^{\text {a }}$ Tested with ANOVA.

Table 3 Age and gender characteristics of 300 statin-treated FH patients with or without new cardiovascular events according to the Taq1B polymorphism

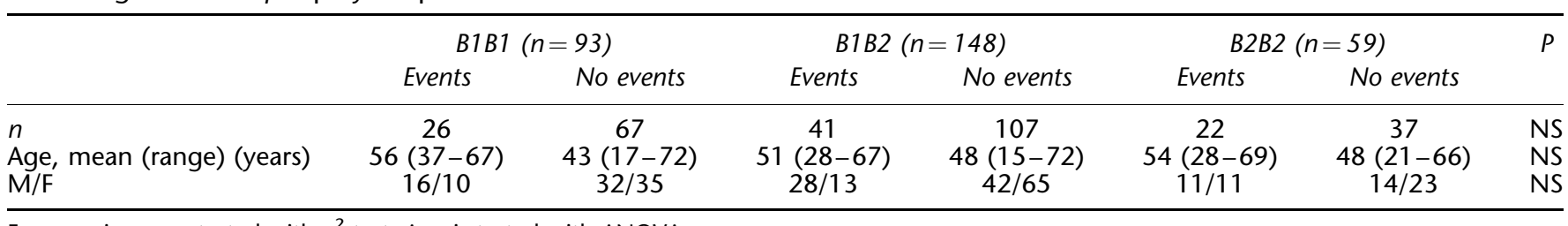

Frequencies were tested with $\chi^{2}$ test. Age is tested with ANOVA.

Comparison was made between the three genotypes in those with or without events.

HDL-cholesterol increased by $10 \%$ upon treatment (all $P<0.0001)$. Mean differences according to genotypes are shown in Table 2. The effect of lipid-lowering medication on plasma T-cholesterol, HDL-cholesterol, and TG concentration was significant in all three genotypes (all $P<0.01$ ) and similar in all three groups.

Lipid levels before and lipid changes after lipid-lowering therapy were similar in FH patients with or without new cardiovascular events in each group (data not shown).

\section{New and recurrent cardiovascular events}

Overall, $30 \% \quad(n=89)$ of the patients developed new cardiovascular events during follow-up. New events were recorded in $60 \%(n=70)$ of the patients with CVD at intake (the 'secondary prevention' group), and in $10 \%(n=19)$ of the patients from the 'primary prevention' group, resembling patients who entered the lipid clinic without a history of CVD. Patients with CVD at intake had a relative risk (RR) of 7.7 (95\% CI 4.3-13.8) for future events. One or more new events were recorded in $37 \%(n=22)$ of the B2B2 subjects, whereas in the carriers of the B1 allele (B1B1 and $\mathrm{B} 1 \mathrm{~B} 2$, respectively) $28 \%(n=67)$ was affected. However, this difference was not significant $(P=0.36)$. There was no significant difference in mean age and sex ratio between the two groups (Table 3 ). In the 67 carriers of the B1 allele, 57 recurrent events occurred, whereas 23 recurrent events were recorded in the $22 \mathrm{~B} 2 \mathrm{~B} 2$ subjects. The total number of events was $124(51 \%)$ and 45 (76\%), respectively, in the B1 allele and B2B2 carriers $\left(\chi^{2}=5.33\right.$; NS).

Figure 1 shows a time-to-first-event analysis according to the three genotypes. B2B2 subjects tended to have a lower event-free survival, compared to the other two genotypes (log rank 2.6; $P=0.1)$.

We compared the RR for CVD for the CETP genotypes, adjusted for age, gender, and CVD at intake. The TaqIB polymorphism appeared to be a significant predictor of future events in a COX regression model. The RR was almost twice as high in subjects with B2B2, compared to $\mathrm{B} 1 \mathrm{~B} 1(\mathrm{RR}=1.8$ (95\% CI: 1.1-3.0)) (Figure 2). This effect of the polymorphism was independent of baseline lipid parameters. After adjusting also for baseline lipid parameters (T-cholesterol, HDL-cholesterol, and TG) in the COX regression model, the effect of the polymorphism became even more significant (RR for CVD events of B2B2 subjects: 2.1 (95\% CI 1.2-3.4)). Although not significant $(P=0.08)$, each $\mathrm{mmol} / \mathrm{l}$ increase in baseline HDL-cholesterol lowered the RR for cardiovascular events with $50 \%$. Using a COX model with lipid parameters after treatment, instead of baseline values, gave similar RR values (RR for B2B2 compared to B1 allele carriers was 1.8).

\section{Discussion}

In the present study, we have assessed the genotype frequencies of the TaqIB polymorphism of the CETP gene in a population of 300 patients with FH. Furthermore, we have investigated the associations of the different alleles with clinical characteristics and lipid profiles in these patients. The frequency of the TaqIB polymorphism in this population was similar to that found in other studies, ${ }^{7,15}$ suggesting that our study population is not genetically different from others. 


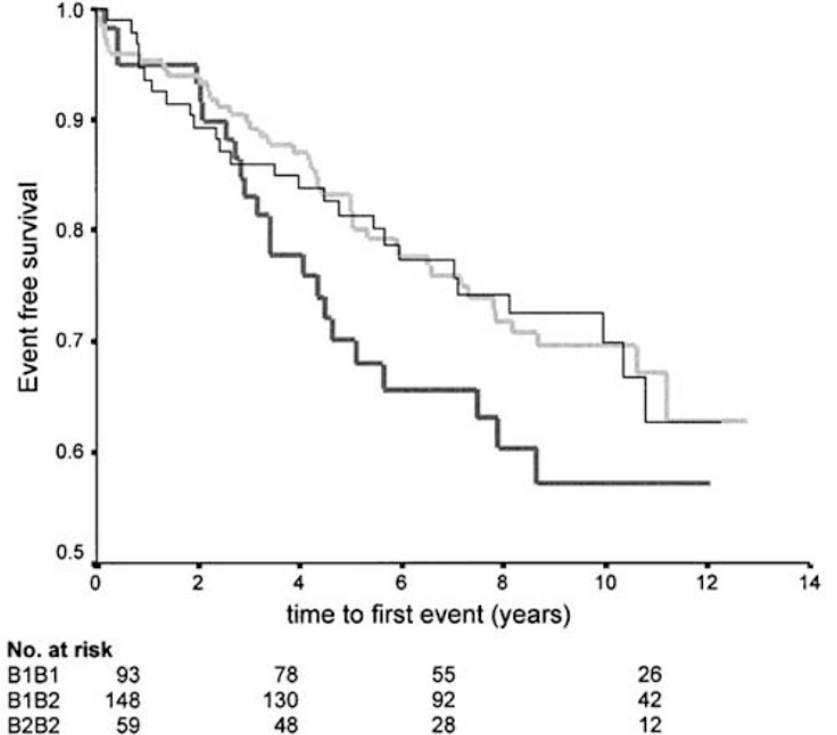

Figure 1 Time-to-event analysis (Kaplan-Meier) for $300 \mathrm{FH}$ patients according to CETP TaqIB polymorphism. Cumulative eventfree survival was poor for B2B2 subjects ( $)$, compared to B1B1 $(\longrightarrow)$ and B1B2 ( $($ ) (log rank 2.6; $P=0.1)$.

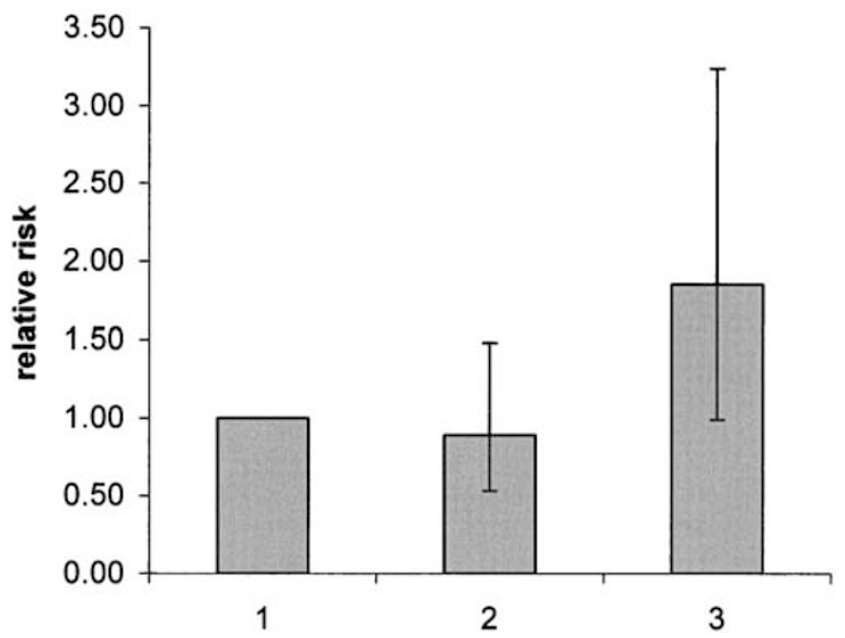

Figure 2 RR for a new cardiovascular event according to CETP TaqIB polymorphism. RR shown with $95 \% \mathrm{CI}$ for a new cardiovascular event in the three different genotypes $(1=\mathrm{B} 1 \mathrm{~B} 1,2=\mathrm{B} 1 \mathrm{~B} 2$, and $3=\mathrm{B} 2 \mathrm{~B} 2)$. The RR is $1.8(95 \% \mathrm{Cl}: 1.1-3.0)$ for $\mathrm{B} 2 \mathrm{~B} 2$ subjects, compared to $\mathrm{B} 1$ allele carriers (COX regression, adjusted for age, gender, and CVD at intake; B1B1 is the reference category).

Our data clearly show an association of the B1 allele of CETP-TaqIB polymorphism with HDL-cholesterol levels at baseline. This association with HDL-cholesterol has been reported by several authors. ${ }^{4-6}$ Other lipid parameters were not significantly different across the TaqIB genotypes. Moreover, we found that B2B2 subjects had an almost doubled risk for developing new cardiovascular events during the follow-up period. Our finding that only 'never smokers' had a significant association of HDL-cholesterol levels with the B1 allele is in line with other studies. ${ }^{4,5,16}$

Despite a more favorable baseline HDL-cholesterol, our patients with the B2B2 genotype had a higher event risk and a poor event-free survival, compared to carriers of the B1 allele. This finding is not in line with other studies that assessed cardiovascular event risks according to this polymorphism, and found that subjects with a B2 allele were more protected. Eiriksdottir et $a l^{8}$ investigated the occurrence of non-fatal MI in men from Iceland, and found that B2B2 subjects had their first MI on average 2 years later than B1 carriers. Durlach et $a l^{17}$ showed that diabetic patients had significantly less coronary heart disease (CHD) when they had the B2B2 genotype, compared to B1 carriers. Furthermore, an odds ratio of 0.7 for $\mathrm{CHD}$ was found in male, but not in female, participants of the Framingham Offspring study. ${ }^{7}$

In recently published studies, higher plasma CETP levels of $\mathrm{FH}$ patients were associated with a more atherogenic lipid profile. ${ }^{18-20}$ In one of these reports, the highest tertile of plasma CETP level was also associated with a higher increase of progression of atherosclerosis after 2 years of statin therapy. ${ }^{20}$ However, higher CETP levels were also associated with a higher body mass index and different sex ratios, which may have confounded these observations. In our study, we found no confounding differences between the three Taq1B genotypes. Even lipid profiles were similar except for the expected higher plasma HDL-cholesterol levels in the B2B2 carriers. Apparently, much of the CETP levels in plasma are determined by other factors than the Taq1B polymorphism. This may explain the observed differences between our study and the other studies in $\mathrm{FH}$ patients.

Our finding of the less favorable outcome in B2B2 subjects is in corroboration with the results of the REGRESS study. ${ }^{15}$ In that study, 807 males with angiographically documented CHD were randomly treated with pravastatin or placebo for 2 years in order to assess the regression of coronary atherosclerosis. Patients with the B1B1 genotype (associated with lower HDL-cholesterol levels) had the strongest progression of coronary atherosclerotic lesions when treated with placebo. However, pravastatin therapy slowed the progression of coronary atherosclerosis in B1B1 carriers but not in B2B2 carriers. The genetic predisposition to the response of statin medication revealed in the study of Kuivenhoven et $a l^{15}$ is confirmed by the results of the present study. This interaction between the Taq1B genotype and statin therapy could not be confirmed in subanalysis studies of two large placebo-controlled trials with pravastatin. ${ }^{21,22}$ At least no additional benefit was found for individuals with the B2B2 genotype. It may be that the effect of the interaction of the Taq1B polymorphism and statin therapy on clinical outcome is specific for FH patients. 
In conclusion, in spite of similar improvement of the lipoprotein profile during statin therapy, our FH patients with the B2B2 genotype may have a higher CVD risk in comparison with the $\mathrm{B} 1$ allele carriers.

\section{References}

1 Gordon T, Castelli WP, Hjortland MC, Kannel WB, Dawber TR: High density lipoprotein as a protective factor against coronary heart disease. The Framingham Study. Am J Med 1977; 62: 707714.

2 Miller GJ, Miller NE: Plasma high-density lipoprotein concentration and development of ischaemic heart disease. Lancet 1975; 1: $16-19$.

3 Koizuma J, Mabuchi H, Yoshimura A et al: Deficiency of serum cholesteryl-ester transfer activity in patients with familial hyperalphalipoproteinaemia. Atherosclerosis 1985; 58: 175-186.

4 Hannuksela ML, Liinamaa MJ, Kesaniemi YA, Savolainen MJ: Relation of polymorphisms in the cholesteryl ester transfer protein gene to transfer protein activity and plasma lipoprotein levels in alcohol drinkers. Atherosclerosis 1994; 110: 35-44.

5 Freeman DJ, Griffin BA, Holmes AP et al: Regulation of plasma HDL cholesterol and subfraction distribution by genetic and environmental factors: associations between the Taq1B RFLP in the CETP gene and smoking and obesity. Arterioscler Thromb 1994; 14: $336-344$.

6 Kuivenhoven JA, de Knijff P, Boer JMA et al: Heterogeneity at the CETP gene locus: influence on plasma CETP concentrations and HDL cholesterol levels. Arterioscler Thromb Vasc Biol 1997; 17: 560-568.

7 Ordovas JM, Cupples A, Corella D et al: Association of cholesteryl ester transfer protein-TaqIB polymorphism with variations in lipoprotein subclasses and coronary heart disease risk. The Framingham Study. Arterioscler Thromb Vasc Biol 2000; 20: $1323-1329$.

8 Eiriksdottir G, Bolla MK, Thorsson B, Sigurdsson G, Humphries SE, Gudnason V: The $-629 \mathrm{C}>$ A polymorphism in the CETP gene does not explain the association of TaqIB polymorphism with risk and age of myocardial infarction in Icelandic men. Atherosclerosis 2001; 159: 187-192.

9 Stone NJ, Levy RI, Frederickson DS, Verter J: Coronary artery disease in 116 kindred with familial type II hyperlipoproteinemia. Circulation 1974; 49: 476-488.

10 Anonymous: Mortality in treated heterozygous familial hypercholesterolemia: implications for clinical management. Scientific Steering Committee on behalf of the Simon Broome Register Group. Atherosclerosis 1999; 142: 105-112.
11 Sijbrands EJG, Westendorp RGJ, Lombardi MP et al: Additional risk factors influence excess mortality in heterozygous familial hypercholesterolemia. Atherosclerosis 2000; 149: 421-425.

12 Mohrschladt MF, Westendorp RGJ, Gevers Leuven JA, Smelt AHM: Cardiovascular disease and mortality in statin-treated patients with familial hypercholesterolemia. Atherosclerosis 2004; 172: 329-335.

13 Friedewald WT, Levy RI, Fredrickson DS: Estimation of the concentration of low-density lipoprotein cholesterol in plasma, without use of the preparative ultracentrifuge. Clin Chem 1972; 18: 499-502.

14 Miller SA, Dykes DD, Polesky HF: A simple salting out procedure for extracting DNA from human nucleated cells. Nucleic Acids Res 1988; 16: $1215-1217$.

15 Kuivenhoven JA, Jukema JW, Zwinderman AH et al: The role of a common variant of the cholesteryl ester transfer protein gene in the progression of atherosclerosis. N Engl J Med 1998; 338: $86-93$.

16 Kondo I, Berg K, Drayna D, Lawn R: DNA polymorphism at the locus for human cholesteryl ester transfer protein (CETP) is associated with high density lipoprotein cholesterol and apolipoprotein levels. Clin Genet 1989; 35: 49-56.

17 Durlach A, Clavel C, Girard-Globa A, Durlach V: Sex-dependent association of a genetic polymorphism of cholesteryl ester transfer protein with high-density lipoprotein cholesterol and macro-vascular pathology in type II diabetic patients. J Clin Endocr Metab 1999; 84: 3656-3659.

18 Carmena-Ramon R, Ascaso JF, Real JT, Najera G, Ordovas JM, Carmena R: Association between the TaqIB polymorphism in the cholesteryl ester transfer protein gene locus and plasma lipoprotein levels in familial hypercholesterolemia. Metabolism 2001; 50: 651-656.

19 Hogue JC, Lamarche B, Gaudet D et al: Relationship between cholesteryl ester transfer protein and LDL heterogeneity in familial hypercholesterolemia. J Lipid Res 2004; 45: 1077-1083.

20 de Grooth GJ, Smilde TJ, van Wissen $\mathrm{S}$ et al: The relationship between cholesteryl ester transfer protein levels and risk factor profile in patients with familial hypercholesterolemia. Atherosclerosis 2004; 173: 261-267.

21 Freeman DJ, Samani NJ, Wilson V et al: A polymorphism of the cholesteryl ester transfer protein predicts cardiovascular events in non-smokers in the West of Scotland Coronary Prevention Study. Eur Heart J 2003; 24: 1833-1842.

22 De Grooth GJ, Zerba KE, Huang SP et al: The cholesteryl ester transfer protein (CETP) Taq1B polymorphism in the Cholesterol And Recurrent Events study (CARE): no interaction with the response to pravastatin therapy and no effects on cardiovascular outcome. J Am Coll Cardiol 2004; 43: 854-857. 